\title{
Quenched binary Bose-Einstein condensates: Spin-domain formation and coarsening
}

\author{
S. De, D. L. Campbell, R. M. Price, A. Putra, Brandon M. Anderson, and I. B. Spielman \\ Joint Quantum Institute, University of Maryland and National Institute of Standards and Technology, College Park, Maryland, 20742, USA
}

(Received 21 November 2012; revised manuscript received 8 January 2014; published 25 March 2014)

\begin{abstract}
We explore the time evolution of two-component Bose-Einstein condensates (BECs), quasi one dimensional with respect to their spinor dynamics, following a quench from one component BECs with a U(1) order parameter into two-component condensates with a $\mathrm{U}(1) \times Z_{2}$ order parameter. In our case, these two spin components have a propensity to phase separate, i.e., they are immiscible. Remarkably, these spin degrees of freedom can equivalently be described as a single-component attractive BEC. A spatially uniform mixture of these spins is dynamically unstable, rapidly amplifying any quantum or pre-existing classical spin fluctuations. This coherent growth process drives the formation of numerous spin-polarized domains, which are far from the system's ground state. At much longer times these domains grow in size, coarsening, as the system approaches equilibrium. The experimentally observed time evolution is consistent with our fully three-dimensional stochastic-projected Gross-Pitaevskii calculation.
\end{abstract}

DOI: 10.1103/PhysRevA.89.033631

PACS number(s): 67.85.Fg, 67.85.De

Ultracold atomic gases are unique systems for studying phase transitions where the full range from adiabatic to diabatic can be easily accessed in the laboratory. A prime example of this is the transition from superfluid (SF) to Mott insulator (MI) in an optical lattice: when the transition is crossed slowly, a nearly $T=0 \mathrm{SF}$ transforms into a nearly $T=0 \mathrm{MI}$ [1]; however, when the system is quenched by rapidly entering the MI regime, it exhibits rapid dynamics before dephasing into a highly excited, high-temperature final state [2-4]. Here we study a similar quantum quench in a two-component spinor BEC, where the spin degree of freedom is initialized in a highly excited state. We follow the resulting dynamics during which spin domains rapidly form, and subsequently slowly relax towards equilibrium as the domain size increases and the domain number decreases.

The establishment of out of equilibrium domains formed by quenching through a phase transition is ubiquitous in physical systems ranging from grain formation in minerals [5], domain nucleation in magnetic systems [6], to Kibble-Zurek phenomena such as structure growth in the early universe [7], and spontaneous vortex formation in quenched BEC's [8]. For an initially zero-temperature system, a quench can result from rapidly traversing a second order quantum phase transition that is associated with a change in the system's symmetry. In the case of a quench from SF to MI, the SF's $\mathrm{U}(1)$ order parameter is absent in the MI phase (with its trivial $\mathrm{Z}_{1}$ order parameter). Another example system is a quenched transverse-field Ising ferromagnet, where the order parameter transforms from $Z_{1}$ to $Z_{2}$ [9]. In our experiment, we prepare a transversely magnetized two-component spinor BEC described by a U(1) order parameter and observe the formation and spatial expansion (coarsening) of domains following a quench into a phase with a $U(1) \times Z_{2}$ order parameter [10,11], unexplored by previous studies with binary

Published by the American Physical Society under the terms of the Creative Commons Attribution 3.0 License. Further distribution of this work must maintain attribution to the author(s) and the published article's title, journal citation, and DOI. condensates (miscible $[12,13]$ or immiscible $[14,15]$ ). As compared with three-component systems [16-22], the relative simplicity present here allows us to identify an intriguing analogy between our spin system and a single-component attractive BEC as it collapses [23-26].

We explore the time-evolving magnetization of twocomponent ${ }^{87} \mathrm{Rb}$ Bose-Einstein condensates (BECs) in the $5 S_{1 / 2}$ electronic ground state. Our BECs are well described in terms of a spinor wave function $\Psi(\mathbf{r})=\left\{\psi_{\uparrow}(\mathbf{r}), \psi_{\downarrow}(\mathbf{r})\right\}$, where the $|\uparrow, \downarrow\rangle$ pseudospins label the $\left|f=1, m_{F}= \pm 1\right\rangle$ atomic spin states. Its dynamics are governed by the spinor Gross-Pitaevskii equation (sGPE)

$$
\begin{aligned}
i \hbar \partial_{t} \psi_{\uparrow, \downarrow}(\mathbf{r})= & {\left[-\frac{\hbar^{2} \nabla^{2}}{2 m}+V(\mathbf{r})+\left(c_{0}-c_{2}\right) n(\mathbf{r})\right.} \\
& \left.+2 c_{2} N\left|\psi_{\uparrow, \downarrow}(\mathbf{r})\right|^{2}\right] \psi_{\uparrow, \downarrow}(\mathbf{r})+\frac{\Omega_{\perp}}{2} \psi_{\downarrow, \uparrow}(\mathbf{r}),
\end{aligned}
$$

a continuum analog to the transverse field Ising model. In Eq. (1), $n(\mathbf{r})=N\left[\left|\psi_{\uparrow}(\mathbf{r})\right|^{2}+\left|\psi_{\downarrow}(\mathbf{r})\right|^{2}\right]$ is the total density, $m$ is the atomic mass, $V(\mathbf{r})$ is a spin-independent external potential, $\Omega_{\perp}$ describes the Zeeman shift of a "transverse" magnetic field, and $c_{0}$ and $c_{2}$ are the spin-independent and spin-dependent interaction coefficients, respectively [27,28]. This Hamiltonian has a $Z_{2}$ symmetry describing a reversal of $|\uparrow\rangle$ and $|\downarrow\rangle$, which is absent in most binary mixtures [12-15]. In the $f=1$ manifold of ${ }^{87} \mathrm{Rb}, c_{0}=100.86 \times 4 \pi \hbar^{2} a_{\mathrm{B}} / m$ vastly exceeds $c_{2} \approx-4.7 \times 10^{-3} c_{0}$, where $a_{\mathrm{B}}$ is the Bohr radius $[29,30]$. For a static density profile and when $\Omega_{\perp}=0$, each spin component in Eq. (1) is separately described by an attractive single-component GPE.

We produce $N=7.0(5) \times 10^{5}$ atom ${ }^{87} \mathrm{Rb}$ BECs [30] in the $\left|f=1, m_{F}=0\right\rangle$ hyperfine state, originating from cold $\mid f=$ $\left.1, m_{F}=-1\right\rangle$ thermal clouds formed in a hybrid magnetic and optical trap [31]. These BECs are subject to a uniform magnetic field with magnitude $B_{0}=107.0(2) \mu \mathrm{T}$ and are confined in the extremely anisotropic crossed optical dipole trap depicted in Fig. 1(a). Our dipole trap is formed from a pair of axially symmetric $1064 \mathrm{~nm}$ laser beams intersecting at right angles with $1 / e^{2}$ radii $\approx 67 \mu \mathrm{m}$ and $\approx 300 \mu \mathrm{m}$. The radial $\left(\mathbf{e}_{r}\right.$, i.e., in the $\mathbf{e}_{x}-\mathbf{e}_{y}$ plane) and axial $\left(\mathbf{e}_{z}\right)$ trap frequencies are 
(a) Schematic

\section{0}

(b) Magnetization

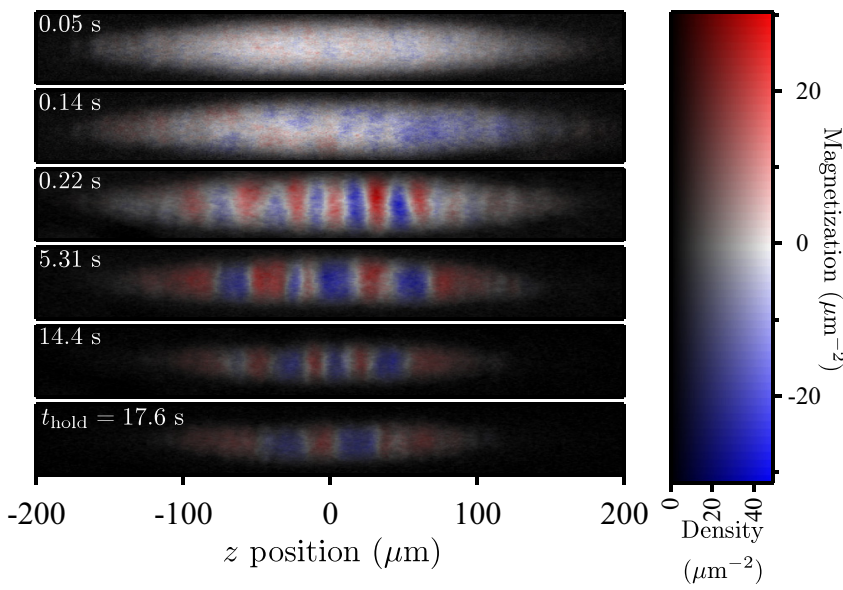

FIG. 1. (Color online) Magnetization $M_{z}(\mathbf{r})$. (a) Schematic illustrating a spinor BEC with domains in an anisotropic crosseddipole trap. (b) Images showing the progression from a uniformly magnetized condensate (short times) in which domains appear (intermediate times) and then grow spatially (long times); during this process the condensate slowly decays away. (c) Color scale indicating the degree of magnetization (colors from blue to red), and the density (intensity from black to colored).

$\omega_{r} / 2 \pi=135(3) \mathrm{Hz}$ and $\omega_{z} / 2 \pi=3.1$ (2) $\mathrm{Hz}$, respectively. Our $T=90(8) \mathrm{nK}$ condensates have radial and axial ThomasFermi radii of $R_{r}=3.9(1) \mu \mathrm{m}$ and $R_{z}=170$ (7) $\mu \mathrm{m}$. The BECs' $170 \mu \mathrm{m}$ axial radius is not small compared to the dipole laser's $300 \mu \mathrm{m}$ waist along the axial direction; as a result, we expect small deviations from the conventional inverted parabola density profile.

Because the typical $c_{0} n(\mathbf{r})$ spin-independent energy vastly exceeds the $c_{2} n(\mathbf{r})$ spin-dependent energy scale, we make the conventional Thomas-Fermi approximation for the overall density distribution $n(\mathbf{r})$ characterized by a chemical potential $\mu$, and a minimum healing length $\xi=\hbar / \sqrt{2 m \mu}$. This gives $n(\mathbf{r})=[\mu-V(\mathbf{r})] /\left[c_{0}+c_{2} M_{z}^{2}(\mathbf{r})\right]$, which depends very weakly on the $z$ component of local magnetization vector, $\mathbf{M}(\mathbf{r})=\left\{M_{x}(\mathbf{r}), M_{y}(\mathbf{r}), M_{z}(\mathbf{r})\right\}=$ $\left\{2 \operatorname{Re}\left[\psi_{\uparrow}^{*}(\mathbf{r}) \psi_{\downarrow}(\mathbf{r})\right], 2 \operatorname{Im}\left[\psi_{\uparrow}^{*}(\mathbf{r}) \psi_{\downarrow}(\mathbf{r})\right],\left|\psi_{\uparrow}(\mathbf{r})\right|^{2}-\left|\psi_{\downarrow}(\mathbf{r})\right|^{2}\right\}$.

The spin degrees of freedom vary almost exclusively with axial, not radial, position [21] because our extremely anisotropic condensate's $\approx 3.9 \mu \mathrm{m}$ radial extent is comparable to the minimum spin healing length $\xi_{s}=\xi\left|c_{0} / c_{2}\right|^{1 / 2}=$ $3.20(4) \mu \mathrm{m}$. Theoretically, we may describe the spin degree of freedom as a one-dimensional (1D) spinor [26] with components $\chi_{\uparrow, \downarrow}(z)=\left|\chi_{\uparrow, \downarrow}(z)\right| e^{i \phi_{\uparrow, \downarrow}(z)}$; retaining terms through first order in $c_{2} / c_{0}$, we obtain an effective 1D sGPE:

$$
i \hbar \partial_{t} \chi_{\uparrow, \downarrow}=\left[-\frac{\hbar^{2} \partial_{z}^{2}}{2 m}-g_{1 \mathrm{D}}(z)+2 g_{1 \mathrm{D}}(z)\left|\chi_{\uparrow, \downarrow}\right|^{2}\right] \chi_{\uparrow, \downarrow} .
$$

The 1D interaction strength $g_{1 \mathrm{D}}(z) \propto c_{2}$ is related to a $1 \mathrm{D}$ healing length $\xi_{1 \mathrm{D}} \approx \sqrt{3 / 2} \xi_{s}$. These two 1D sGPEs are coupled by the local constraints $\left|\chi_{\uparrow}(z)\right|^{2} \partial_{z} \phi_{\uparrow}(z)+\left|\chi_{\downarrow}(z)\right|^{2} \partial_{z} \phi_{\downarrow}(z)=0$ (i.e., no mass currents in our experiment). To make the analogy explicit, we dropped terms quadratic in $\left|\chi_{\uparrow, \downarrow}\right|^{2}$ resulting from integrating out the transverse dimensions. These repulsive terms do not affect the dynamics at short times after the quench but must be included at long times.

Our spinor experiment is initiated by a $34 \mu$ s rf pulse that puts each atom into a equal-amplitude superposition of the $|\uparrow, \downarrow\rangle=\left|m_{F}= \pm 1\right\rangle$ spin states, the ground state when $\Omega_{\perp}$ is large; the system then evolves according to Eq. (1) with $\Omega_{\perp}=0$. This procedure is equivalent to rapidly quenching $\Omega_{\perp}$ to zero: the ground state goes from breaking a U(1) symmetry to breaking a different $\mathrm{U}(1)$ symmetry along with a $Z_{2}$ symmetry. While a conventional BEC breaks just a single $\mathrm{U}(1)$ symmetry associated with a wave function's overall phase (generated by the identity), our spinor Hamiltonian adds a U(1) symmetry associated with the relative phase of the spin (generated by the Pauli matrix $\check{\sigma}_{z}$ ), as well as a discrete $Z_{2}$ symmetry. Post quench, the formation of spin domains corresponds to breaking the $Z_{2}$ symmetry, while within a specific domain, a new $U(1)$ symmetry is broken. This is generated by a combination of the overall and relative phases: each spin domain has a broken generator $\left(\check{1} \pm \check{\sigma}_{z}\right) / 2$, leaving behind a "sneaky" unbroken U(1) symmetry generated by $\left(\check{1} \mp \check{\sigma}_{z}\right) / 2$.

The quenched binary mixture is held for a variable duration $t_{\text {hold }}$, up to $20 \mathrm{~s}$, while spin structure forms and evolves. Spinmixing collisions are suppressed because the relatively large $82 \mathrm{~Hz}$ quadratic Zeeman shift greatly exceeds the $c_{2} n(\mathbf{r}) \approx$ $6 \mathrm{~Hz}$ spin-dependent energy [16]. As a result, we observe no population in $m_{F}=0$ for the entire duration of our experiment. After $t_{\text {hold }}$, we remove the confining potential and allow the atomic ensemble to expand (largely transversely) for $19.3 \mathrm{~ms}$, during which time we Stern-Gerlach [32] separate the spin components. We detect the resulting density distribution by absorption imaging and reconstruct both $M_{x}(x, z)$ and $M_{z}(x, z)$, projected onto the $\mathbf{e}_{z}-\mathbf{e}_{x}$ imaging plane. A brief rf pulse just before time of flight (TOF) can partially repopulate $\mid m_{F}=$ $0\rangle$; following TOF expansion and Stern-Gerlach separation, the distribution of all three spin states contains sufficient information to obtain $M_{x}$ and $M_{z}$ simultaneously. We depict representative reconstructions of $M_{z}(x, y)$ at six hold times in Fig. 1(b).

The initially $\left(t_{\text {hold }}=0\right)$ uniform $\chi(z)=(|\uparrow\rangle+|\downarrow\rangle) / \sqrt{2}$ spin superposition is dynamically unstable, as indicated in the snapshots in Fig. 1(b). At this unstable point, small spin-wave excitations have an $\left(\hbar \omega / \mu_{1 \mathrm{D}}\right)^{2}=\left(k \xi_{1 \mathrm{D}}\right)^{2}\left[\left(k \xi_{1 \mathrm{D}}\right)^{2}-2\right]$ energy spectrum [22], where $\mu_{1 \mathrm{D}}=\hbar^{2} /\left(2 m \xi_{1 \mathrm{D}}^{2}\right)$ is a typical 1D spin-interaction energy. When $\hbar \omega$ is imaginary-for $k \xi_{1 \mathrm{D}} \in$ $(0, \sqrt{2})$ - the associated modes grow exponentially with peak gain at $k=1 / \xi_{1 \mathrm{D}}$, amplifying any existing spin fluctuations, classical or quantum. Figure 2 depicts the magnetization $M_{z}(z)$, showing the initially unmagnetized condensate develop visible structure after about $200 \mathrm{~ms}$. The experimental data plotted in Fig. 2(a) are in qualitative agreement with a stochastic-projective GPE (SP-GPE) simulation [33], with parameters nearly matched to our experiment, Fig. 2(b). 

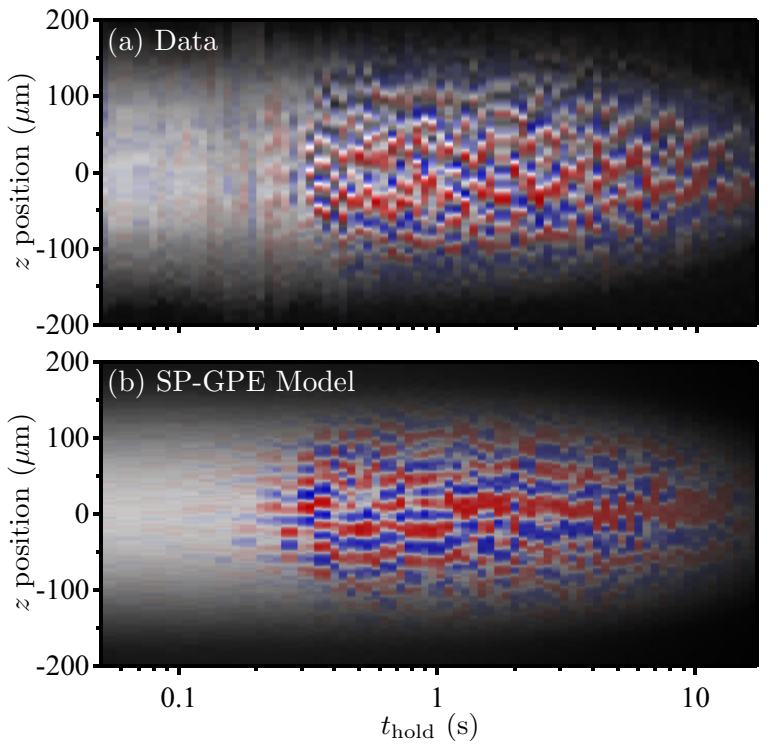

FIG. 2. (Color online) Time evolution of magnetization $M_{z}(z)$. (a) Experimental data and (b) finite-temperature simulation using the SP-GPE method. In both simulation and experiment, the spatial structure of $M_{z}(z)$ coarsens after an initial growth period as domains coalesce.

In what follows, we make several quantitative comparisons between the two. The SP-GPE's stochastic noise term was chosen to match the experimentally observed temperature and was not tuned to match the onset time for domain formation. While the amplitude of these spin waves grow with an exponential time constant $\tau(k)=1 / \operatorname{Im}(\omega(k, z))$ [minimum at $\left.\tau(z)=2 m \xi_{\mathrm{DD}}^{2}(z) / \hbar \approx 42 \mathrm{~ms}\right]$, Fig. 2 shows that no structure is visible until $t_{\text {hold }} \approx 200 \mathrm{~ms}$.

Our simulations predict that structure begins to grow immediately; however, the domains that have formed directly after the quench cannot be detected due to the magnetization $M_{z}(z)$ at the beginning of the exponential formation process being smaller in magnitude than the technical noise (primarily due to shot noise in density fluctuations across the BEC) in the system. The methodology of detecting a spin domain in the BEC is based upon detecting the number of modulations in $M_{z}(z)$ that are larger in amplitude than the measured noise in $M_{z}(z)$. After the quench, when the magnetization of the domains grows rapidly in amplitude, there is a threshold once domains will become statistically measurable in the analysis. This measurement effect is evident in the rapid increase of domains we experimentally detect in Fig. 3 at $\approx 200 \mathrm{~ms}$.

Figure 2 also shows that spin structure forms more slowly in the lower-density periphery of the system where $\xi_{1 \mathrm{D}}$ and $\tau$ are larger. To quantify this effect, Fig. 3 plots the number of spin regions visible above the noise, along with the results of our SP-GPE simulations, and a local-density-approximation (LDA, accounting for our system's inhomogeneous density profile) prediction for the expected pattern of domain growth. This number increases for short times because spin regions become visible in the system's center before its edges and do not initially reflect a change of their spatial size.

The spin modulations continue to grow in amplitude until, at $t_{\text {hold }} \approx 300 \mathrm{~ms}$, they form fully spin polarized domains of $|\uparrow\rangle$,

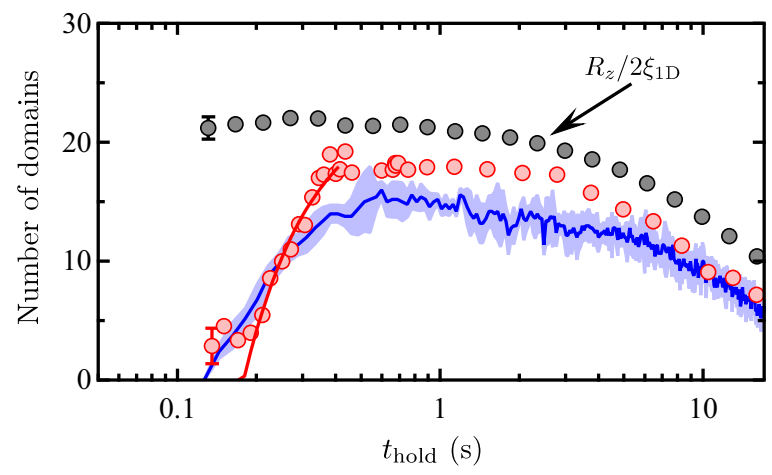

FIG. 3. (Color online) Number of domains as a function of $t_{\text {hold }}$. The red symbols depict the experimentally observed number of domains (typical uncertainty plotted on the leftmost point) and the blue curve plots the results of our SP-GPE simulation (uncertainties denoted by the blue band). In both cases, the uncertainties reflect the standard deviation over many realizations. In addition, the red curve fits the data to a model assuming exponential growth along with a nonzero observation threshold, in the LDA. The gray symbols correspond to the ratio $R_{z} /\left(2 \xi_{1 \mathrm{D}}\right)$ : an estimate of domain number, assuming the system with length $2 R_{z}$ is partitioned into domains of local size $\pi \xi_{1 \mathrm{D}}(z)$ (the size at which domains initially form); the weighted average of this over our system is about $4 \xi_{1 D}$.

and $|\downarrow\rangle$, with a spacing set by the dynamic growth process, not by the system's equilibrium thermodynamics. After this period of rapid growth, the polarized spin domains evolve slowly, equilibrating, for the remaining $2 \mathrm{~s}$ duration of our experiment.

Our BEC has a $\tau=10(1) \mathrm{s}$ lifetime, implying that the domain pattern must evolve in time as the BEC slowly contracts. The simplest model-in which the domain pattern contracts together with the dwindling BEC (where each domain simply contracts) —is obviated by Fig. 3, that shows the number of domains decreasing after $t_{\text {hold }} \approx 1 \mathrm{~s}$. Indeed, once a domain becomes smaller than $\approx 2 \xi_{1 \mathrm{D}}(z)$, it can no longer reach full spin polarization in its center, and it ceases to be a barrier for the hydrodynamic flow of the other spin state. As a result, small domains de-pin and can move freely until they coalesce with another domain of the same spin.

While Figs. 2 and 3 qualitatively suggest that the domains gradually expand as $t_{\text {hold }}$ increases from $300 \mathrm{~ms}$ to $20 \mathrm{~s}$, it is difficult to obtain a quantitative measure of domain size from data in this form. Indeed, the data show that while measurements at neighboring times have similar domain sizes, the exact domain pattern has a significant element of randomness-primarily in the form of phase shifts-likely resulting from subtle differences in the initial conditions, as amplified by the subsequent exponential gain process. To mitigate these effects, we turn to the power spectral density $\operatorname{PSD}_{x, z}(k)=\left|\int M_{x, z}(z) \exp (i k z) d z\right|^{2}$ obtained from these data. With the PSD, we can compare different realizations even in the presence of spatial phase shifts of the domain structure.

Figure 4(a) shows $\operatorname{PSD}_{z}(k)$ derived from $M_{z}(z)$ shown in Fig. 2. For short times $\left(t_{\text {hold }} \lesssim 300 \mathrm{~ms}\right)$, a narrow peak associated with the growing spin modulations develops. Once the spin domains reach unity polarization, the magnetization's magnitude saturates and the boundaries between domains- 


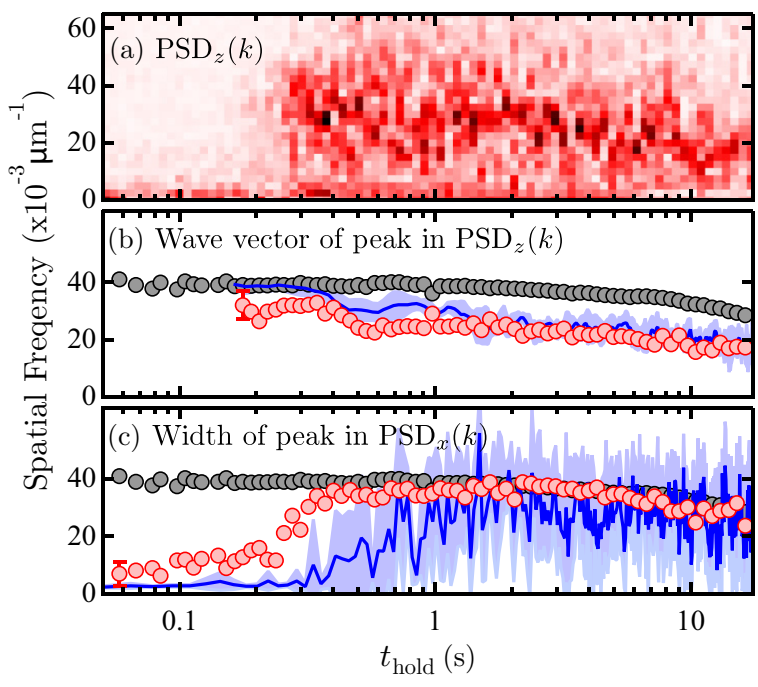

FIG. 4. (Color online) Power spectral density (PSD). (a) $\operatorname{PSD}_{z}(k)$ as a function of $t_{\text {hold }}$ showing the formation of a peak at finite wave vector $k$, followed by the gradual movement of that peak to smaller $k$ as the spin domains expand. Each vertical slice represents a single experimental realization, i.e., no averaging. The color scale depicts increasing spectral power with darker color. (b) Wave-vector of peak of $\operatorname{PSD}_{z}(k)$. (c) Width of $\operatorname{PSD}_{x}(k)$, which always peaked around zero. In panels (b) and (c), the red symbols depict the experimentally observed peak location (typical uncertainty plotted on the leftmost point) and the blue curve plots the results of our SP-GPE simulation (uncertainties denoted by the blue band). In these three cases, the uncertainties reflect the standard deviation over eleven realizations, i.e., panels (b) and (c) show averaged data. The gray symbols mark $1 / \xi_{1 \mathrm{D}}$, the homogenous-system wave vector of maximum gain (the uncertainties are comparable to the symbol size).

domain walls - sharpen, broadening $\operatorname{PSD}_{z}(k)$ starting at $t_{\text {hold }} \approx$ $250 \mathrm{~ms}$. At longer times, the broad peak drifts to smaller wave vector, indicating an increasing typical domain size. Figure 4(b) compares this peak location for both experiment and theory against $1 / \xi_{1 \mathrm{D}}$. Figure 4 plots experimental data with red symbols and the SP-GPE simulation with the blue curve. Our analytical model predicts maximum gain at this wave vector and indeed our SP-GPE simulation shows peak gain at $1 / \xi_{1 \mathrm{D}}$. By contrast, the peak in $\operatorname{PSD}_{z}(k)$ for the experiment is at slightly smaller $k$.

Because the $\approx 2 \xi_{1 \mathrm{D}}(z)$ minimum domain size increases as the condensate depletes away, it is plausible that the increase in domain size results exclusively from an increasing cutoff in the minimum domain size. If we assume a proportional relationship between $1 / \xi_{1 \mathrm{D}}(z)$ (Fig. 4, gray symbols) and the peak in $\operatorname{PSD}_{z}(k)$ such that the ratio of one over the other would hold constant, we see in Fig. 4(b) that for $t_{\text {hold }}>2 \mathrm{~s}$ this theory could describe the data. To highlight this possible relationship, we display the ratio between $\operatorname{PSD}_{z}(k)$ and $1 / \xi_{1 D}(z)$-essentially constant - on a linear time scale in Fig. 5 (the uncertainties reflect the standard deviation of the mean at each $\left.t_{\text {hold }}\right)$.

Unlike $\operatorname{PSD}_{z}(k), \operatorname{PSD}_{x}(k)$ is peaked about zero. This is because $M_{x}(z)$ is only appreciable in the domain walls where

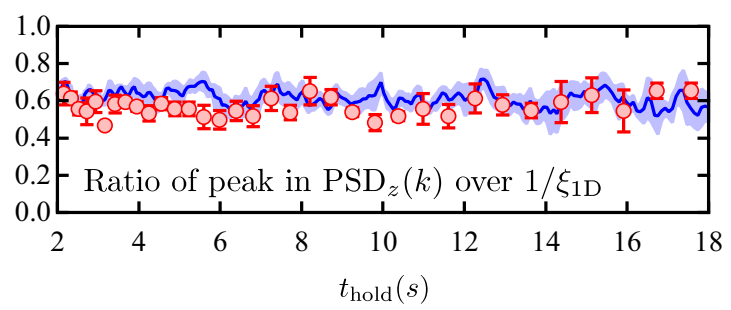

FIG. 5. (Color online) Ratio of peak in $\operatorname{PSD}_{z}(k)$ over $1 / \xi_{1 \mathrm{D}}$ plotted on a linear time scale. We use only the seven data runs that include $t_{\text {hold }}>6 \mathrm{sec}$. Unlike the rest of the paper, the uncertainties given here represent the standard deviation of the mean. The red symbols depict the experimentally observed peak location and the blue curve shows the results of our SP-GPE simulation (uncertainties denoted by the blue band).

the gas is not fully polarized; it consists of a series of narrow peaks. By showing that the width of the peak in $\operatorname{PSD}_{x}(k)$ tracks the inverse spin-healing length, Fig. 4(c) demonstrates that the domain walls are sized according to $\xi_{1 \mathrm{D}}$ (gray symbols).

In experiments, we saw a small, but repeatable large-scale structure in $M_{z}(z)$ due to a very small residual gradient $\partial_{z} B=$ $\gamma z$, where $\gamma=0.092$ (4) $\mathrm{G} / \mathrm{cm}^{2}$. Although small, we could further mitigate the effects of this contribution with a spin echo $\pi$ pulse, flipping between $|\downarrow\rangle$ and $|\uparrow\rangle$ midway between the quench and the beginning of TOF. This removed the large-scale spin structure from the inhomogeneous magnetic fields but left the spin dynamics - which were associated with much shorter length scales - otherwise unaffected. Furthermore, well above our ambient gradients, a counterflow instability [13] seeds spin-structure growth.

For $c_{2}<0$, as in ${ }^{87} \mathrm{Rb}$, Eqs. (1) and (2) describe our system's spin degree of freedom as a single-component attractive BEC (the overall density follows the conventional Thomas-Fermi profile). The process of domain formation is a spinor analog to the "chain of pearls" pattern that forms in 1D BECs quenched from repulsive to attractive interactions [23,24]. In that case, the growth of structure results from a modulational instability with peak gain at $k=1 / \xi$ set by the conventional healing length. Attractive Bose systems are intrinsically unstable against collapse [25]; however, for spinors, any eventual collapse is stymied by an effective hard-core interaction resulting from the bounded individual spin wave functions and higher-order interaction terms omitted from Eq. (2).

Thus, we observe the full gamut of time scales starting with the dynamical generation of spin-domains from an initially nonequilibrium system followed by their subsequent relaxation to progressively larger domains, i.e., coarsening. However, for times $>2 \mathrm{~s}$ this coarsening occurs in lock step with the only other relevant length scale: the spin healing length.

We appreciate discussions with and terminology introduced by N. Bray-Ali, and insight gleaned from C. Raman. We acknowledge the financial support the NSF through the Physics Frontier Center at JQI, and the ARO with funds from both the Atomtronics MURI and DARPA's OLE Program. 


\section{APPENDIX: STOCHASTIC-PROJECTIVE GROSS-PITAEVSKII EQUATION}

The numerical simulations were performed using the stochastic-projective Gross-Pitaevskii version of Eq. (1) with $\Omega_{\perp}=0$ :

$$
\begin{aligned}
d \psi_{\mathbf{C}}(\mathbf{r}, t)= & \mathcal{P}_{\mathbf{C}}\left\{\frac{\gamma(t)}{k_{B} T(t)}\left(\mu(t)-L_{\mathbf{C}}\right) \psi_{\mathbf{C}}(\mathbf{r}, t) d t-\frac{\psi_{\mathbf{C}}(\mathbf{r}, t)}{2 \tau} d t\right. \\
& \left.-\frac{i}{\hbar} L_{\mathbf{C}} \psi_{\mathbf{C}}(\mathbf{r}, t) d t+d W_{\gamma}(\mathbf{r}, t)\right\},
\end{aligned}
$$

where

$$
\begin{aligned}
L_{c}= & -\frac{\hbar^{2} \nabla^{2}}{2 m}+V(\mathbf{r})+c_{0} N_{0}\left(\left|\psi_{\mathbf{C} \uparrow}\right|^{2}+\left|\psi_{\mathbf{C} \downarrow}\right|^{2}\right) \\
& +c_{2} N_{0}\left(\left|\psi_{\mathbf{C} \uparrow}\right|^{2}-\left|\psi_{\mathbf{C} \downarrow}\right|^{2}\right) \sigma_{z},
\end{aligned}
$$

and the stochastic noise term $d W_{\gamma}(\mathbf{r}, t)$ satisfies

$$
\left\langle d W_{\gamma}^{*}(\mathbf{r}, t) d W_{\gamma}\left(\mathbf{r}^{\prime}, t\right)\right\rangle=\frac{2 \gamma(t) d t}{N_{0}} \delta\left(\mathbf{r}-\mathbf{r}^{\prime}\right) .
$$

The parameters of the noise and damping terms are varied slowly to keep them consistent with the thermodynamic quantities describing the BEC. To account for atom loss, the total atom number was assumed to satisfy $N(t)=N_{0} e^{-t / \tau}$, where $\tau=10 \mathrm{~s}$ is the timescale for atom loss. To phenomenologically account for observed cooling, the temperatures was adjusted as $T(t)=T_{f}+\left(T_{i}-T_{f}\right) e^{-t / 3.5 \mathrm{~s}}$ where $T_{i}=90 \mathrm{nK}$ and
$T_{f}=70 \mathrm{nK}$. The chemical potential $\mu(t)$ is then calculated using $N(t)$ and $T(t)$ for the experimental trap geometry. The noise term $\gamma(t)$ was then calculated according to Eq. (172) in Ref. [33].

The simulation is performed in two parts. First, the initial $m_{F}=0$ thermal condensate is modeled using a singlecomponent SP-GPE with atom number $N(0)$ and temperature $T_{i}$. The system is prepared in a state $\psi_{0}$ by evolving the singlecomponent SP-GPE with $c_{2}=0$ for a time $|t| \gg 1 / \gamma(0)$. At $t=0$, a two-component SP-GPE is evolved using Eq. (A2) for initial conditions $\psi_{\uparrow}=\psi_{\downarrow}=\psi_{0} / \sqrt{2}$. This simulates the effects of the rf-pulse transforming the initial $m_{F}=0$ state into a superposition of $m_{F}= \pm 1$ states. For this simulation, the atom number and temperature are varied as described above.

The calculation is performed using a full three-dimensional simulation for a system of size $L_{x}=L_{y}=11 \mu \mathrm{m}$ and $L_{z}=416 \mu \mathrm{m}$ on a grid with $N_{x}=N_{y}=24$ and $N_{z}=768$. Evolution was performed using the split-operator method with the cutoff performed using a momentum-space grid. The experimental apparatus has an estimated imaging resolution of $\sim 2 \mu \mathrm{m}$, which is larger than the resolution of the simulation. To avoid spurious domain counting, the density profiles were filtered with a low-pass filter to remove spatial modes with resolution smaller than the experiment. The data analysis performed for the SP-GPE was then identical to that of the experiment. To account for the stochastic behavior of the SP-GPE, five identical simulations were performed.
[1] M. Greiner, O. Mandel, T. Esslinger, T. Hänsch, and I. Bloch, Nature (London) 415, 39 (2002).

[2] M. Greiner, O. Mandel, T. W. Hansch, and I. Bloch, Nature (London) 419, 51 (2002).

[3] J. Sebby-Strabley, B. L. Brown, M. Anderlini, P. J. Lee, W. D. Phillips, J. V. Porto, and P. R. Johnson, Phys. Rev. Lett. 98, 200405 (2007).

[4] S. Will, T. Best, U. Schneider, L. Hackermuller, D.-S. Luhmann, and I. Bloch, Nature (London) 465, 197 (2010).

[5] A. Putnis, An Introduction to Mineral Sciences (Cambridge University Press, New York, 1992).

[6] A. Hubert and R. Schäfer, Magnetic Domains (Springer, Berlin, 1998), Vol. 21.

[7] T. W. B. Kibble, J. Phys. A: Math. Gen. 9, 1387 (1976).

[8] C. N. Weiler, T. W. Neely, D. R. Scherer, A. S. Bradley, M. J. Davis, and B. P. Anderson, Nature (London) 455, 948 (2008).

[9] P. Calabrese, F. H. L. Essler, and M. Fagotti, J. Stat. Mech.: Theory Exp. (2012) P07016.

[10] R. Barnett, A. Turner, and E. Demler, Phys. Rev. Lett. 97, 180412 (2006).

[11] Y. Kawaguchi and M. Ueda, Phys. Rev. A 84, 053616 (2011).

[12] D. M. Weld, P. Medley, H. Miyake, D. Hucul, D. E. Pritchard, and W. Ketterle, Phys. Rev. Lett. 103, 245301 (2009).

[13] M. A. Hoefer, J. J. Chang, C. Hamner, and P. Engels, Phys. Rev. A 84, 041605 (2011).

[14] D. S. Hall, M. R. Matthews, J. R. Ensher, C. E. Wieman, and E. A. Cornell, Phys. Rev. Lett. 81, 1539 (1998).
[15] K. M. Mertes, J. W. Merrill, R. Carretero-González, D. J. Frantzeskakis, P. G. Kevrekidis, and D. S. Hall, Phys. Rev. Lett. 99, 190402 (2007).

[16] J. Stenger, S. Inouye, D. M. Stamper-Kurn, H. J. Miesner, A. P. Chikkatur, and W. Ketterle, Nature (London) 396, 345 (1998).

[17] W. Zhang, D. L. Zhou, M.-S. Chang, M. S. Chapman, and L. You, Phys. Rev. Lett. 95, 180403 (2005).

[18] L. E. Sadler, J. M. Higbie, S. R. Leslie, M. Vengalattore, and D. M. Stamper-Kurn, Nature (London) 443, 312 (2006).

[19] M. Vengalattore, J. Guzman, S. R. Leslie, F. Serwane, and D. M. Stamper-Kurn, Phys. Rev. A 81, 053612 (2010).

[20] J. Guzman, G.-B. Jo, A. N. Wenz, K. W. Murch, C. K. Thomas, and D. M. Stamper-Kurn, Phys. Rev. A 84, 063625 (2011).

[21] E. M. Bookjans, A. Vinit, and C. Raman, Phys. Rev. Lett. 107, 195306 (2011).

[22] D. M. Stamper-Kurn and M. Ueda, Rev. Mod. Phys. 85, 1191 (2013).

[23] K. E. Strecker, G. B. Partridge, A. G. Truscott, and R. G. Hulet, Nature (London) 417, 150 (2002).

[24] S. E. Pollack, D. Dries, M. Junker, Y. P. Chen, T. A. Corcovilos, and R. G. Hulet, Phys. Rev. Lett. 102, 090402 (2009).

[25] E. A. Donley, N. R. Claussen, S. L. Cornish, J. L. Roberts, E. A. Cornell, and C. E. Wieman, Nature (London) 412, 295 (2001).

[26] Z. Dutton and C. W. Clark, Phys. Rev. A 71, 063618 (2005).

[27] T.-L. Ho, Phys. Rev. Lett. 81, 742 (1998). 
[28] T. Ohmi and K. Machida, J. Phys. Soc. Jpn. 67, 1822 (1998).

[29] E. G. M. van Kempen, S. J. J. M. F. Kokkelmans, D. J. Heinzen, and B. J. Verhaar, Phys. Rev. Lett. 88, 093201 (2002).

[30] All uncertainties herein reflect the uncorrelated combination of single-sigma statistical and systematic uncertainties.
[31] Y.-J. Lin, A. R. Perry, R. L. Compton, I. B. Spielman, and J. V. Porto, Phys. Rev. A 79, 063631 (2009).

[32] W. Gerlach and O. Stern, Z. Phys. A 9, 349 (1922).

[33] P. B. Blakie, A. S. Bradley, M. J. Davis, R. J. Ballagh, and C. W. Gardiner, Adv. Phys. 57, 363 (2008). 